\title{
Performance of Logistic Model Tree Classifier using Statistical Features for Fault Diagnosis of Single Point Cutting Tool
}

\author{
M. Ramesh Nachiappan ${ }^{1 *}$, V. Sugumaran' and M. Elangovan ${ }^{2}$ \\ 'School of Mechanical and Building Sciences (SMBS), VIT University, Chennai Campus, Chennai - 600127, Tamil \\ Nadu, India; ramesh.manoharan@outlook.com, v_sugu@yahoo.com \\ 2Department of Mechanical Engineering, Amrita School of Engineering, Amrita Vishwa Vidyapeetham, \\ Amrita University, Coimbatore - 641112,Tamil Nadu, India; elango59@yahoo.in
}

\begin{abstract}
Objective: A variety of tool condition monitoring techniques in modern manufacturing system plays a key role in estimating the tool wear which can save the machine downtime and increase the cutting tool utilization. Tool wear compromises dimensional accuracy and affects the precision, tolerance and surface finish. An active condition monitoring system of tool health is required for superior productivity. Method/Analysis: In this experimental study, the accelerometer was used to acquire the vibration signal during the turning operation in a lathe machine with good and fault simulated single point cutting tool. The signals are acquired for all combinations of spindle speeds, feed rates, depth of cuts and tool wear level. In this study, 3 different spindle speeds, feed rates and depth of cuts, and 4 different tool wear levels were considered. Statistical features were extracted from the acquired signal and substantial features were recognized using a decision tree algorithm. The identified substantial statistical features were considered in classifying signals using logistic model tree classifier. Findings: The classification accuracy obtained for all the signals combined (i.e., variable spindle speeds, feed rates, depth of cuts and tool wear levels) were found to be $74.27 \%$. The classification accuracy achieved was improved through simplifying the model by considering feed rate and depth of cut as variable factor. The accuracy of the classification was found to be in the range of $82-86 \%$. Further, the classification accuracy was found to increase to the range of $82-93 \%$, when considering the depth of cut alone as variable factor. Application/Improvement: The utilization of logistic model tree to identify the tool wear level in a single point cutting tool during turning operation was comprehensively analysed in this study. The performance of the classifier on fault diagnosis of single point cutting tool and its improvement by reducing the complexity of the model was discussed.
\end{abstract}

Keywords: Decision Tree, Feature Extraction, Logistic Model Tree, Logistic Regression, Statistical Features Tool Condition Monitoring

\section{Introduction}

The manufacturing industries play a significant part in building the economy of under-developed and emerging nations. Tool condition monitoring (TCM) is an emerging area which promises production automation and increased productivity. We are trailing towards automation era, where automation has started to occupy the shop floors in the manufacturing industry than ever before. We need an intelligent system which can monitor the health of the cutting tool. Most commonly observed wear in machining is flank wear and it is an unescapable phenomenon in the metal cutting operation. The consequences of machining with a worn cutting tool consist of dimensional inaccuracy, increased length of the machining process, and effect on the tolerance of the assembly, surface finish and moreover the machine. Therefore,

${ }^{*}$ Author for correspondence 
a thorough learning of diagnosing the tool wear at the prompt stage is required. Engineers and researchers are struggling to develop a matured condition monitoring system for cutting tools. In ${ }^{1}$ discussed many different techniques for each phase of tool condition monitoring. The phases include sensing technique selection, extraction of features, feature reduction and feature classification. $\operatorname{In}^{2}$ used cutting force, acoustic emission and vibration sensing techniques to assess the state of tool during turning operation. $\operatorname{In}^{3}$ reviewed the applications of using digital imaging as sensing technique for tool condition monitoring. $\mathrm{In}^{4}$ analysed the signals of turning operation with many different types of features extracted from the time domain and also frequency domain. The various features extracted by them in their experiments were various wavelet, statistical and frequency features. $\operatorname{In}^{\frac{5}{5}}$ discussed the technique of feature reduction to categorize the highly contributing features using a decision tree algorithm in estimation of remaining useful bearing life. $\operatorname{In}^{6}$ utilized the features extracted from Gray-Level Co-Occurrence Matrix (GLCM) of machined surface images to classify different tool states. $\operatorname{In}^{7}$ discussed the effectiveness diagnosing the fault in single point cutting tool using decision tree approach. In ${ }^{8}$ discussed the optimization techniques to improve the decision tree algorithm. The dimensionality reduction of the data was done using feature selection approach. Further the decision tree's performance was found to be improved with cross validation and reduced error pruning. $\mathrm{In}^{2}$ used $\mathrm{v}$ support vector machine $(\gamma$ -SVM) to recognize different tool wears. $\operatorname{In}^{10}$ compared the effectiveness of support vector machines, artificial neural networks, kernel logistic regression, and logistic model tree algorithms in predicting the landslide. In this study, vibration signals are used to monitor the condition of the cutting tool. Statistical features obtained from the vibration signals were utilized in the classification. The decision tree algorithm is used for feature reduction.

\section{Experimental Arrangement}

The experimental study was carried out in a lathe machine. Figure 1 and Figure 2 shows the experimental arrangement consisting of accelerometer, cutting tool, specimen, data acquisition device and laptop. The specimens used in the experiment were $25 \mathrm{~mm}$ diameter mild steel bar. The cutting tool used in the experiment is a brazed carbide tipped single point cutting tool. Dytran Uniaxial accelerometer was used to record the vibration signal from machining process. The accelerometer was secured over the cutting tool with the aid of glue. The accelerometer output was connected to a National Instruments USB data acquisition device NIVIB 4432. The analog signal output from the accelerometer is converted into digital signal by the data acquisition device, which can be understood by the computer software. The capturing of the digital signal was done using National Instruments LabVIEW software. Table 1 shows the various machining parameters considered for the turning operation. The vibration signal was acquired for all combinations of the spindle speed, feed rate, and depth of cut with four different levels of flank wear.

Table 1. Variable process parameters

\begin{tabular}{|l|l|l|l|l|l|}
\hline Variables & \multirow{2}{*}{ Unit } & \multicolumn{4}{|l|}{ Levels } \\
\cline { 3 - 6 } & & $\mathbf{1}$ & $\mathbf{2}$ & $\mathbf{3}$ & $\mathbf{4}$ \\
\hline Spindle speed & $\mathrm{rpm}$ & 510 & 770 & 900 & \\
\hline Feed rate & $\mathrm{mm} / \mathrm{rev}$ & 0.109 & 0.122 & 0.135 & \\
\hline Depth of cut & $\mathrm{mm}$ & 0.5 & 0.8 & 1.0 & \\
\hline Flank wear & $\mathrm{mm}$ & 0 & 0.2 & 0.4 & 0.6 \\
\hline
\end{tabular}

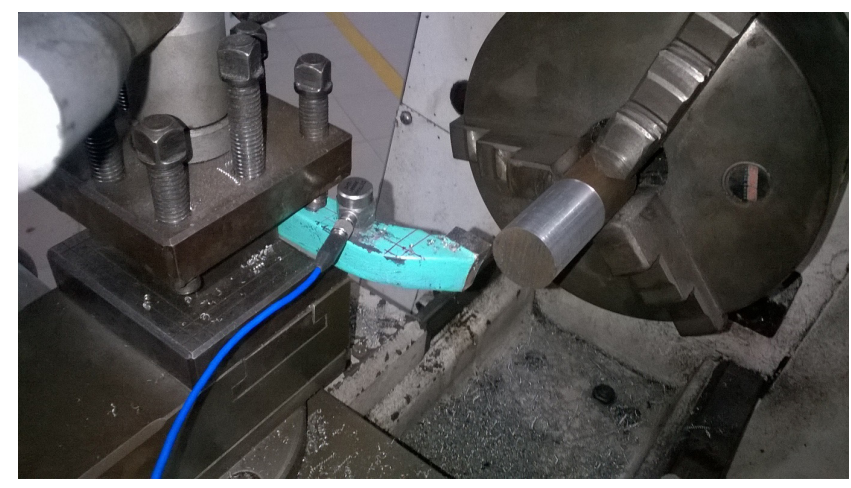

Figure 1. Experimental setup (accelerometer, cutting tool and specimen).

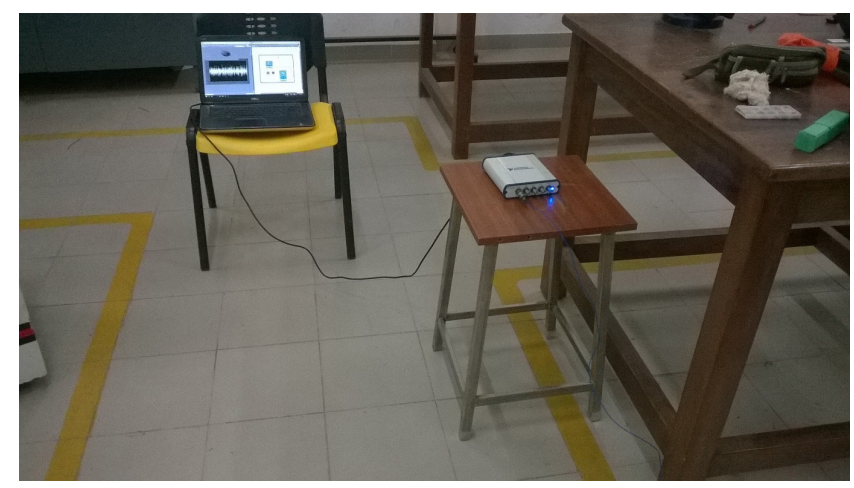

Figure 2. Experimental setup (data acquisition device and computer). 


\subsection{Experimental Procedure}

\subsubsection{Acquisition of Baseline Signal}

The uniaxial accelerometer was placed securely over the single point cutting tool as shown in Figure 1. The peak frequency observed in the system was $6 \mathrm{kHz}$. The sampling frequency of the system is taken as $20 \mathrm{kHz}$ since the Nyquist sampling theorem states that the sampling frequency of the system must be at least two times the peak frequency observed in the system. The specimen was fixed in the chuck firmly and rough turning operation was done preliminarily to eliminate the layers of oxides formed over the surface and to level surface of the specimen equally. First few seconds of the turning operation after rough turning operation were not recorded persistently in order to neglect random variation in the signal. After the stabilization of the turning operation, acceleration signals were recorded using the data acquisition system for different machining parameters sequentially.

\subsubsection{Fault Simulation}

The flank wear was introduced in the cutting tool using 'tool and cutter grinder' manually. Lines were drawn for reference parallel to the tangent of the nose radius of the single point cutting tool. Before inducing flank wear to the cutting tool, the length between the topmost point of the nose radius and the line of reference were noted down. After inducing the wear, the length between the new topmost point and the line of reference was measured again. The wear level of the cutting tool is given by finding the difference between the two measurements.

\subsubsection{Acquisition of Acceleration Signal}

The vibration of the cutting tool while machining was acquired from the accelerometer, once the turning operation gets steadied. The sampling frequency of the accelerometer was fixed to $20 \mathrm{kHz}$ and the sample length of the signal was set to 2000 points for all machining conditions. Figure 3 to Figure 6 shows the time-domain plots taken from the cutting tool for various wear levels.

\section{Feature Extraction}

The statistical features taken into consideration in this study include mean, standard error, variance, median, standard deviation, mode, skewness, kurtosis, mini- mum, maximum, range, and sum. The time domain data of acceleration signals is used to extract the above mentioned twelve statistical features. All the features may not have the necessary data for the classification. Those features which are not supporting the classification can be ignored. The detailed explanation of the procedure is given in the feature reduction. Here C4.5 algorithm was used to reduce the unwanted features.

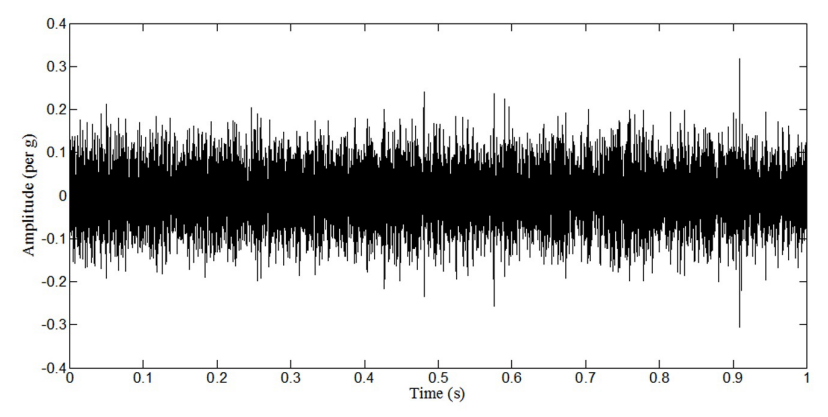

Figure 3. Time domain plot of good tool signal.

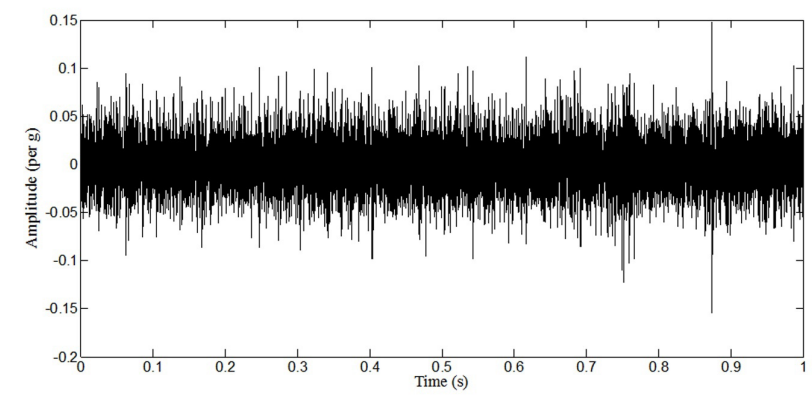

Figure 4. Time domain plot of $0.2 \mathrm{~mm}$ worn tool signal.

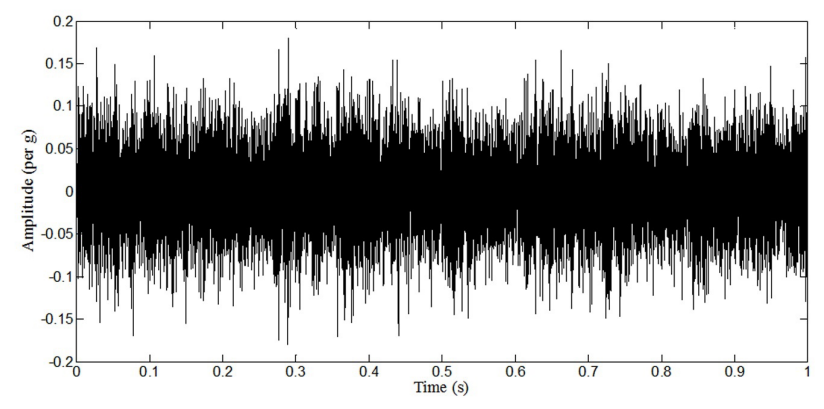

Figure 5. Time domain plot of $0.4 \mathrm{~mm}$ worn tool signal.

\section{1 eature Reduction using Decision Tree}

The extracted twelve statistical features were used as input for the J48 decision tree algorithm. The contribution of 
each feature can be recognized from their position in the tree. The feature which contains more information about the classification will be at the uppermost level of the decision tree, whereas the feature which does not contain any or very less information about the classification will be at the lowermost level. The removal of features which has very less information about the classification can improve the classification accuracy and also save computing time.

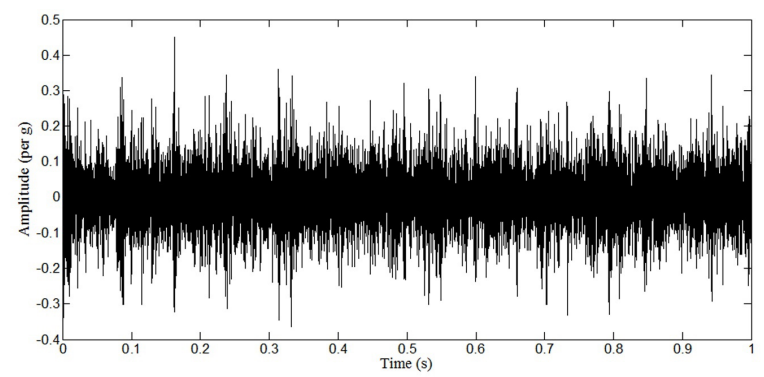

Figure 6. Time domain plot of $0.6 \mathrm{~mm}$ worn tool signal.

\section{Classifier Description}

\subsection{Logistic Model Tree}

Logistic model trees (LMTs) combine two complementary classifiers, linear logistic regression and tree induction. The performance of LMTs are competitive to many powerful classifiers like boosted decision trees. The interpretation of this tree is very simple. The structure of the decision tree contains logistic regression functions at the leaves. Based on the threshold value, the leaf node will be divided into two child nodes where the branch to the right will contain the value of the attribute greater than the threshold and the branch to the left will contain the value of the attribute lesser than the threshold. It is shown in Figure 7. Logit boost algorithm is the commonly used to fix the threshold. Logit boost uses a group of functions $F_{k}$ to forecast classes $1, \ldots, k$ by means of weak learners ${ }^{M}$.

$$
F_{k}(x)=\sum_{m=1}^{k} f_{m k}(x)
$$

\subsection{Decision Tree}

Decision tree is a knowledge design approach where trees are used to exemplify classification rules. J48 is a decision tree classifier which uses $\mathrm{C} 4.5$ algorithm to generate the decision tree. The $\mathrm{C} 4.5$ algorithm creates recursive partitioning of data to form a decision tree. The depth first approach is used to grow decision here. A typical decision tree comprises one root, numerous branches, leaves, and nodes. A decision is taken in the decision tree at every node. The selection of the most noteworthy feature for classification can be found using appropriate estimation criteria. The standards involved in choosing the best feature is based on the concept of information gain and entropy reduction. Information gain is the predicted decrease in the entropy due to separating samples according to the specified attribute. Entropy is the categorization of impurities of a random collection of occurrences. By adding additional information, the ambiguity can be reduced. Information gain evaluates the alteration in entropy beforehand and after adding information to the system. Information gain $(\boldsymbol{s}, \boldsymbol{x})$ of a feature $\boldsymbol{x}$ relative to piling of instances $s$ is given by,

$\operatorname{Gain}(s, x)=\operatorname{Entropy}(s)-\sum_{v \in \text { Value }(x)} \frac{\left|s_{v}\right|}{|s|} \operatorname{Entropy}\left(s_{v}\right)$

Where $\operatorname{Value}(x)$ is the group of all possible values for attribute $x$ and $s_{v}$ is the subset of $s$ for which feature $x$ has value of $v$. Entropy(s) in the equation indicates the entropy of the original group $s$ and

$\sum_{v \in \text { Value }(x)} \frac{\left|s_{v}\right|}{|s|} \operatorname{Entropy}\left(s_{v}\right)_{\text {indicates the predicted }}$ value of the entropy after $s$ is separated using the feature $^{x}$. Entropy is the amount of irregularity of the set of occurrences.

Entropy $(s)=\sum_{i=1}^{n}-p_{i} \cdot \log _{\mathbf{2}} p_{i}$

Where $p_{i}$ is the partition of $s$ belonging to class $i$ and ${ }^{n}$ is the number of classes.

\section{Results and Discussions}

The study on classification of single point cutting tool condition with logistic model tree classifier is discussed in the coming sections as follows:

- Choosing the noteworthy features to ease the computing effort of the classification i.e., Feature reduction. 
- Classification accuracy of logistic model tree classifiers.

- Justification of logistic model tree classifier.

\subsection{Feature Reduction}

The reduction of extracted features was done using J48 decision tree classifier. The classification accuracy of $71.11 \%$ was observed for the $\mathrm{J} 48$ decision tree classifier. The decision tree contains 680 leaves and size of the tree was $1359.72 .86 \%$ classification accuracy was observed with reduced error pruning in $\mathrm{J} 48$ decision tree. The tree had 186 leaves and size of the tree was 371 . When enhancing the minimum number of objects in the classification, the classification accuracy was observed to be $72.31 \%$ with 34 leaves and size of the tree as 67 . Table 2 shows the influence of features in classification accuracy. The features which are not listed in the Table 2 does not contain adequate information for classification. Therefore, those unwanted features can be removed for further calculation. Thereby, reducing the computing effort required to classify the signal. The maximum classification accuracy was witnessed from the graph shown in Figure 8 with five features. The five features which contributed for the maximum classification accuracy was taken for the further classification.

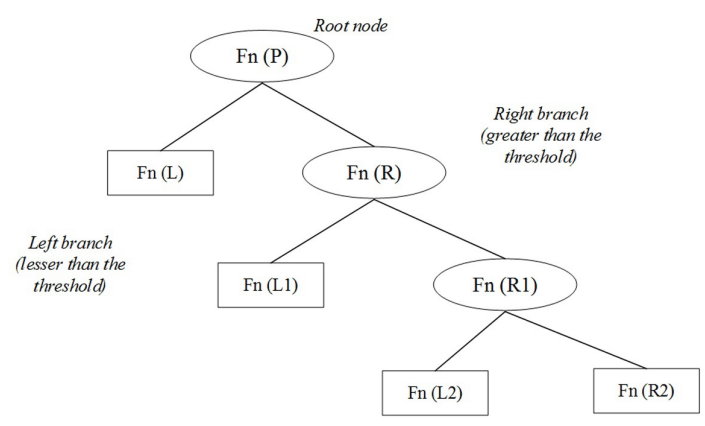

Figure 7. Structure of logistic model tree.

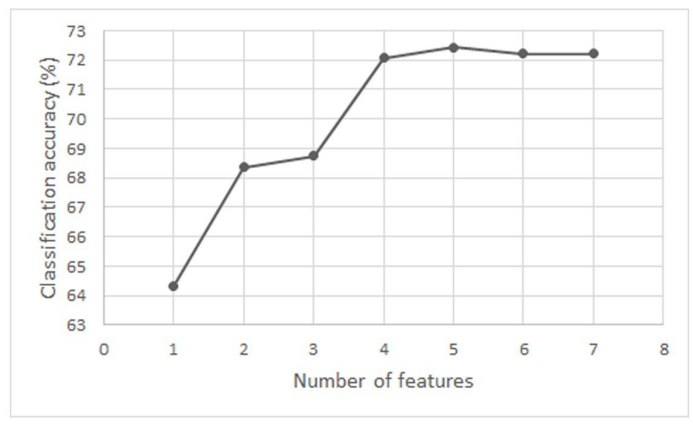

Figure 8. Number of features vs. classification accuracy.
Table 2. Feature combination and their classification accuracy

\begin{tabular}{|l|l|l|}
\hline $\begin{array}{l}\text { No. of } \\
\text { features }\end{array}$ & Features & $\begin{array}{l}\text { Classification } \\
\text { accuracy (\%) }\end{array}$ \\
\hline 1 & Standard deviation & 64.34 \\
\hline 2 & $\begin{array}{l}\text { Standard deviation + } \\
\text { skewness }\end{array}$ & 68.35 \\
\hline 3 & $\begin{array}{l}\text { Standard deviation + } \\
\text { skewness + variance }\end{array}$ & 68.74 \\
\hline 4 & $\begin{array}{l}\text { Standard deviation + } \\
\text { skewness + variance + sum }\end{array}$ & 72.07 \\
\hline 5 & $\begin{array}{l}\text { Standard deviation + } \\
\text { skewness + variance + sum + } \\
\text { kurtosis }\end{array}$ & 72.42 \\
\hline 6 & $\begin{array}{l}\text { Standard deviation + } \\
\text { skewness + variance + sum }+ \\
\text { kurtosis + range }\end{array}$ & 72.21 \\
\hline 7 & $\begin{array}{l}\text { Standard deviation + } \\
\text { skewness + variance + sum }+ \\
\text { kurtosis + range + maximum }\end{array}$ & 72.21 \\
\hline
\end{tabular}

\subsection{Classification Accuracy of Logistic Model Tree Classifier}

The classification accuracy of the logistic model tree (LMT) classifier for the collection of all the 108 experiments signal is observed to be $74.27 \%$. To increase the accuracy of classification, the intricacy can be reduced by taking spindle speed as a distinct factor. The classification accuracy of LMT classifier is given in Table 3 and the comparison is shown in Figure 9. By considering spindle speed as a distinct factor provides an increase of $8-12 \%$, on contrast with the preceding classification of all signals combined. To additionally increase the classification accuracy, the intricacy can be reduced by taking spindle speed and feed rate as distinct factors. The classification accuracy of the LMT classifier for this case is given in Table 4 and the comparison is shown in Figure 10. By considering spindle speed and feed rate as distinct factors provides an increase of $8-19 \%$, on contrast with the classification of all signals combined. For a few combinations of spindle speed and feed rate, the classification accuracy was observed to be lesser than the value achieved in the classification with spindle speed as a distinct factor. To additionally increase the classification accuracy, the intricacy can be reduced by taking the spindle speed, feed rate and depth of cut as distinct factors. The classification accuracy of the LMT classifier for this case is given in Table 5 and the comparison is shown in Figure 11. By 
considering spindle speed, feed rate and depth of cut as distinct factors provides an increase of $8-25 \%$, on contrast with the classification of all signals combined. When considering many separate models, the classification accuracy intensifies. As the number of model increases, the time for setting up the condition monitoring system also increases. The logistic model tree for all signals combined is shown in Figure 12.

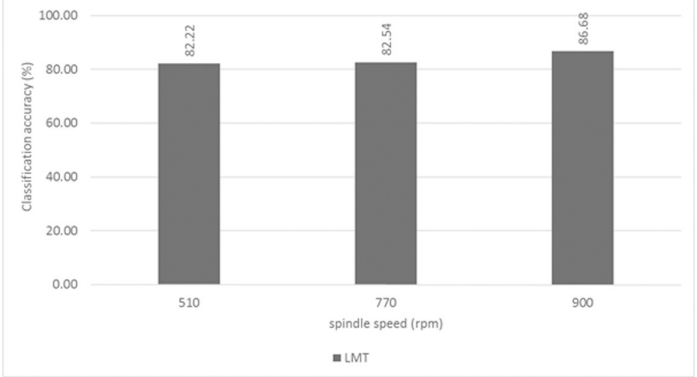

Figure 9. Comparison of classification accuracy without considering depth of cut and feed rate as separate factors.

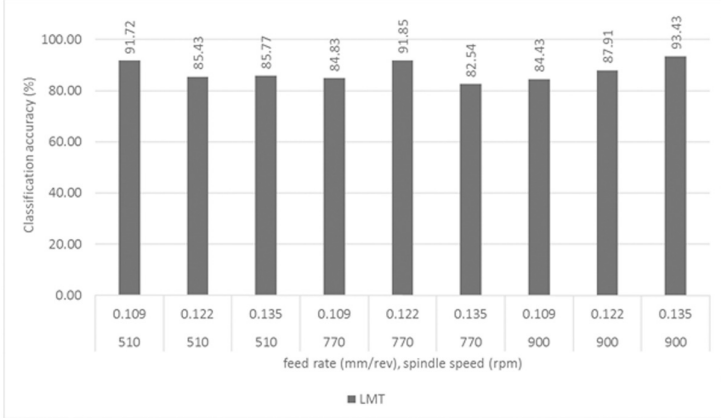

Figure 10. Comparison of classification accuracy without considering depth of cut as a separate factor.

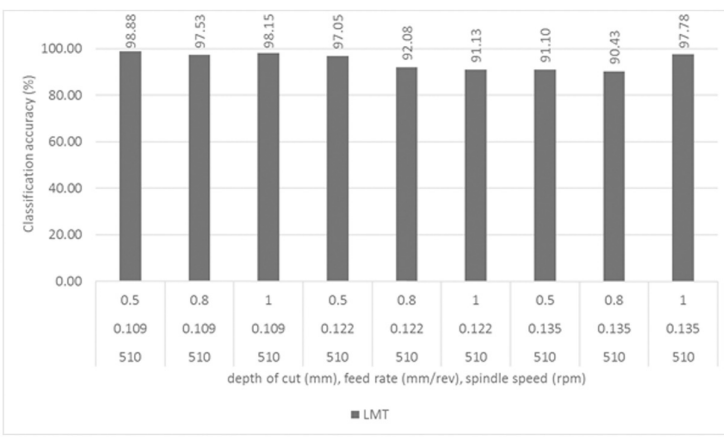

Figure 11. Comparison of classification accuracy of every signal with $510 \mathrm{rpm}$ spindle speed.

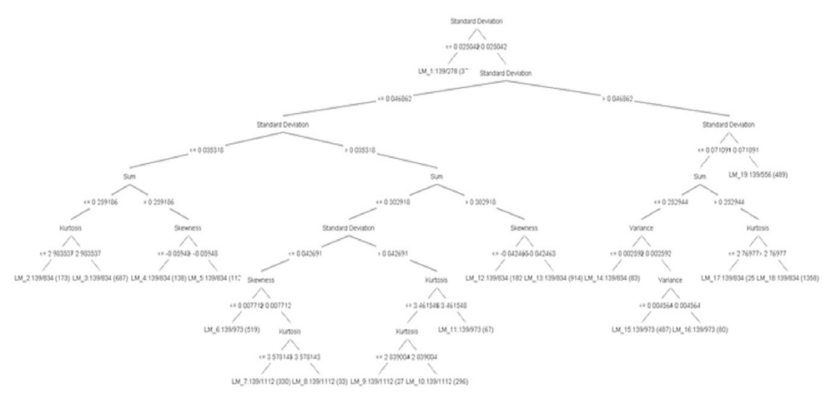

Figure 12. Logistic model tree for all signals combined.

Table 3. Classification accuracy of LMT without considering feed rate and depth of cut as distinct factors

\begin{tabular}{|l|l|}
\hline spindle speed $(\mathrm{rpm})$ & Classification accuracy of LMT (\%) \\
\hline 510 & 82.22 \\
\hline 770 & 82.54 \\
\hline 900 & 86.68 \\
\hline
\end{tabular}

Table 4. Classification accuracy of LMT without considering depth of cut as a separate factor

\begin{tabular}{|l|l|l|}
\hline $\begin{array}{l}\text { spindle } \\
\text { speed }(\mathrm{rpm})\end{array}$ & $\begin{array}{l}\text { Feed rate } \\
(\mathrm{mm} / \mathrm{rev})\end{array}$ & $\begin{array}{l}\text { Classification accuracy of } \\
\text { LMT }(\%)\end{array}$ \\
\hline 510 & 0.109 & 91.72 \\
\hline 510 & 0.122 & 85.43 \\
\hline 510 & 0.135 & 85.77 \\
\hline 770 & 0.109 & 84.83 \\
\hline 770 & 0.122 & 91.85 \\
\hline 770 & 0.135 & 82.54 \\
\hline 900 & 0.109 & 84.43 \\
\hline 900 & 0.122 & 87.91 \\
\hline 900 & 0.135 & 93.43 \\
\hline
\end{tabular}

\subsection{Classifier Validation}

The interpretation of the confusion matrix is described here for the further discussion. Refer the confusion matrix shown in Figure 13, the foremost row of the confusion matrix point to the good condition of the cutting tool. The number of signals which are of good condition and characterized as good is denoted by the first element of the confusion matrix. The second element of the confusion matrix denotes the number of signals which are of good 
condition and characterized as $0.2 \mathrm{~mm}$ wear (FLW1). Likewise, the third element in the confusion matrix denotes the number of signals which are of good condition and characterized as $0.4 \mathrm{~mm}$ wear (FLW2). The fourth element of the confusion matrix denotes the number of signals which are of good condition and characterized as $0.6 \mathrm{~mm}$ wear (FLW3). The second row of the confusion matrix point to $0.2 \mathrm{~mm}$ wear of the cutting tool. Here, the second element in the second row of the confusion matrix is the correctly characterized instances of FLW1. Likewise, the interpretation can be stretched to remaining elements of the confusion matrix. The correctly classified instances are given by the diagonal elements and the misclassified instances are given by the off diagonal elements in the confusion matrix. It can be observed from the confusion matrix that many instances of GOOD condition are misclassified as FLW2 and FLW3. The misclassification could be due to the signals recorded for some machining parameters are similar to signals of FLW2 and FLW3 conditions. Very less number of instances were misclassified in case of FLW1 condition. High misclassification is observed among FLW2 and FLW3 conditions. The misclassification could be due to the difference in flank wear level between FLW2 and FLW3 condition is $0.2 \mathrm{~mm}$. The cutting edge for both FLW2 and FLW3 conditions might have been alike, which lead to the similarity of FLW2 and FLW3 signals.

Table 5. Classification accuracy of LMT considering depth of cut, feed rate and spindle speed as distinct factors

\begin{tabular}{|l|l|l|l|}
\hline $\begin{array}{l}\text { spindle } \\
\text { speed }(\mathrm{rpm})\end{array}$ & $\begin{array}{l}\text { Feed rate } \\
(\mathrm{mm} / \mathrm{rev})\end{array}$ & $\begin{array}{l}\text { Depth } \\
\text { of cut } \\
(\mathrm{mm})\end{array}$ & $\begin{array}{l}\text { Classification } \\
\text { accuracy of LMT } \\
(\%)\end{array}$ \\
\hline 510 & 0.109 & 0.5 & 98.88 \\
\hline 510 & 0.109 & 0.8 & 97.53 \\
\hline 510 & 0.109 & 1 & 98.15 \\
\hline 510 & 0.122 & 0.5 & 97.05 \\
\hline 510 & 0.122 & 0.8 & 92.08 \\
\hline 510 & 0.122 & 1 & 91.13 \\
\hline 510 & 0.135 & 0.5 & 91.10 \\
\hline 510 & 0.135 & 0.8 & 90.43 \\
\hline 510 & 0.135 & 1 & 97.78 \\
\hline 770 & 0.109 & 0.5 & 83.90 \\
\hline 770 & 0.109 & 0.8 & 99.88 \\
\hline 770 & 0.109 & 1 & 89.65 \\
\hline
\end{tabular}

\begin{tabular}{|l|l|l|l|}
\hline 770 & 0.122 & 0.5 & 97.00 \\
\hline 770 & 0.122 & 0.8 & 99.33 \\
\hline 770 & 0.122 & 1 & 99.03 \\
\hline 770 & 0.135 & 0.5 & 82.33 \\
\hline 770 & 0.135 & 0.8 & 92.15 \\
\hline 770 & 0.135 & 1 & 89.30 \\
\hline 900 & 0.109 & 0.5 & 88.23 \\
\hline 900 & 0.109 & 0.8 & 93.20 \\
\hline 900 & 0.109 & 1 & 98.55 \\
\hline 900 & 0.122 & 0.5 & 93.68 \\
\hline 900 & 0.122 & 0.8 & 98.80 \\
\hline 900 & 0.122 & 1 & 97.63 \\
\hline 900 & 0.135 & 0.5 & 99.13 \\
\hline 900 & 0.135 & 0.8 & 94.75 \\
\hline 900 & 0.135 & 1 & 98.63 \\
\hline
\end{tabular}

\begin{tabular}{|c|c|c|c|c|}
\hline GOOD & FLW1 & FLW2 & FLW3 & $<$ classified as \\
\hline 2067 & 3 & 394 & 236 & GOOD \\
\hline 5 & 2399 & 113 & 183 & FLW1 \\
\hline 296 & 187 & 1836 & 381 & FLW2 \\
\hline 350 & 165 & 460 & 1725 & FLW3 \\
\hline
\end{tabular}

Figure 13. Confusion matrix of LMT classifier for all signals combined.

\section{Conclusion}

The effort for this study is to check the performance and aptness of logistic model tree classifier for single point cutting tool condition monitoring. Extracted descriptive statistical features were reduced using J48 decision tree algorithm to lessen the computing effort required to classify the signal. The classification accuracy of all signals combined for logistic model tree classifier was found to be $74.27 \%$. This model would be suitable for machining where multiple combinations of spindle speed, feed rate and depth of cut will be used for a job. If only different depth of cut is involved in machining a job, then a separate model could be created for it. The classification accuracy obtained here ranges between $82 \%$ and $93 \%$. If only different depth of cut and feed rate is involved in machining a job, the classification accuracy ranges between $82 \%$ and $86 \%$. If the operator can modify the settings of condition monitoring system for each combination of spindle speed, feed rate and depth of cut, then the classification accuracy obtained ranges between $82 \%$ and $100 \%$. 


\section{References}

1. Marwala T. Condition monitoring using computational intelligence methods: applications in mechanical and electrical systems. Springer London. 2012.

2. Segreto T, Simeone A, Teti R. Multiple sensor monitoring in nickel alloy turning for tool wear assessment via sensor fusion. Procedia CIRP. 2013 Oct; 12:85-90.

3. Dutta S, Pal SK, Mukhopadhyay S, Sen R. Application of digital image processing in tool condition monitoring: A review. CIRP Journal of Manufacturing Science and Technology. 2013 Apr; 6(3):212-32.

4. Jemielniak K, Urbanski T, Kossakowska J, Bombinski S. Tool condition monitoring based on numerous signal features. The International Journal of Advanced Manufacturing Technology. 2012 Mar; 59(1):73-81.

5. Satishkumar R, Sugumaran V. Estimation of Remaining Useful Life of Bearings based on Support Vector Regression. Indian Journal of Science and Technology. 2016 Mar; 9(10):1-7.

6. Bhat NN, Dutta S, Vashisth T, Pal S, Pal SK, Sen R. Tool condition monitoring by SVM classification of machined surface images in turning. The International Journal of Advanced Manufacturing Technology. 2016 Apr; 83(9):1487-502.

7. Gangadhar N, Kumar H, Narendranath S, Sugumaran V. Fault Diagnosis of Single Point Cutting Tool through Vibration Signal using Decision Tree Algorithm. Procedia Materials Science. 2014 Sep; 5:1434-41.

8. Galathiya AS, Ganatra AP, Bhensdadia CK. Improved Decision Tree Induction Algorithm with Feature Selection, Cross Validation, Model Complexity and Reduced Error Pruning. International Journal of Computer Science and Information Technologies. 2012; 3(2):3427-31.

9. Wang GF, Yang YW, Zhang YC, Xie QL. Vibration sensor based tool condition monitoring using $v$ support vector machine and locality preserving projection. Sensors and Actuators A: Physical. 2014 Mar; 209:24-32.

10. Bui DT, Tuan TA, Klempe H, Pradhan B, Revhaug I. Spatial prediction models for shallow landslide hazards: a comparative assessment of the efficacy of support vector machines, artificial neural networks, kernel logistic regression, and logistic model tree. Landslides. 2016 Apr; 13(2):361-78. 